EPJ Web of Conferences 35, 02004 (2012)

DOI: $10.1051 /$ epjconf/20123502004

(C) Owned by the authors, published by EDP Sciences, 2012

\title{
Development of SiPM-based scintillator tile detectors for a multi-layer fast neutron tracker
}

\author{
R. Preston ${ }^{1,2}$, J. Jakubek ${ }^{3}$, D. Prokopovich ${ }^{4}$, and J. Uher ${ }^{5}$ \\ ${ }^{1}$ CSIRO, Process Science and Engineering \& Minerals Down Under Research Flagship, Lucas Heights, Australia \\ ${ }^{2}$ University of Wollongong, Centre for Medical Radiation Physics, Wollongong, Australia \\ ${ }^{3}$ Czech Technical University, Institute of Experimental and Applied Physics, Prague, Czech Republic \\ ${ }^{4}$ Australian Nuclear Science and Technology Organisation, Institute of Materials Engineering, Lucas Heights, Australia \\ ${ }^{5}$ Amsterdam Scientific Instruments, Amsterdam, The Netherlands
}

\begin{abstract}
We are developing thin tile scintillator detectors with silicon photomultiplier (SiPM) readout for use in a multi-layer fast-neutron tracker. The tracker is based on interleaved Timepix and plastic scintillator layers. The thin $15 \times 15 \times 2 \mathrm{~mm}$ plastic scintillators require suitable optical readout in order to detect and measure the energy lost by energetic protons that have been recoiled by fast neutrons. Our first prototype used dual SiPMs, coupled to opposite edges of the scintillator tile using light-guides. An alternative readout geometry was designed in an effort to increase the fraction of scintillation light detected by the SiPMs. The new prototype uses a larger SiPM array to cover the entire top face of the tile. This paper details the comparative performance of the two prototype designs. A deuterium-tritium (DT) fast-neutron source was used to compare the relative light collection efficiency of the two designs. A collimated UV light source was scanned across the detector face to map the uniformity. The new prototype was found to have 9.5 times better light collection efficiency over the original design. Both prototypes exhibit spatial non-uniformity in their response. Methods of correcting this non-uniformity are discussed.
\end{abstract}

\section{Introduction}

Fast neutron detection often relies on measuring the energy deposited in a detector by elastically recoiled nuclei. As fast neutrons undergo elastic collisions with the target nuclei, the kinetic energy transferred to the nucleus depends on the angle of recoil, with the remaining kinetic energy carried away by the scattered neutron.

In order to measure the energy and direction of an incoming neutron, more sophisticated techniques are required. Under development is a multi-layer detector, based on Timepix pixellated semiconductor detectors interleaved with thin plastic scintillator tiles [1, 2]. The plastic scintillators provide a hydrogenous target in which fast neutrons may elastically collide with hydrogen nuclei (protons), with some recoiling protons escaping the plastic and being measured by the neighbouring Timepix detector. Timepix is a single-quantum counting pixellated silicon detector [3]. In the tracker it operates in time-over -threshold mode, where the charge deposited in each individual pixel is measured. A map of the ionisation track of the proton is produced, which allows its direction and energy on entry to the detector to be determined. By measuring information at multiple scattering points, the original incident neutron's energy and direction may be calculated [4].

With suitable optical readout, the scintillator can provide two additional functions; to generate a trigger for the accompanying Timepix detector acquisition, and to measure the energy lost by the escaping protons in the scintillator volume, which can be used to improve reconstruction resolution. This paper describes work to optimise the design of the plastic scintillator component of the tracker.

Two different readout geometries are compared, with the aim in improving the proportion of scintillation photons reaching the SiPM active area. This quantity is described as the light collection efficiency (LCE).

\section{Detector design and readout}

The tile detector to be used in the multi-layer detector has a number of unique design requirements. The thin tile has dimensions of $15 \times 15 \times 2 \mathrm{~mm}$. The $2 \mathrm{~mm}$ thickness is sufficiently small that a proportion of recoiling protons are able to escape the volume of the scintillator. The lower $15 \times 15 \mathrm{~mm}$ side facing the Timepix cannot be painted or have a reflective wrapping, as this would

This is an Open Access article distributed under the terms of the Creative Commons Attribution License 2.0, which permits unrestricted use, distribution, and reproduction in any medium, provided the original work is properly cited. 
degrade the energy of, or completely stop, escaping protons.

The silicon photomultiplier (SiPM), being suitably compact and capable of measuring small light pulses, was identified as an appropriate detector for the optical readout of the tile detector [5]. The SiPM is a solid-state device based on a matrix of avalanche photodiode subpixels operating in a self quenched Geiger-mode [6, 7]. These sub-pixels are connected in parallel to form a pseudo-analogue photo-detector. Peak photo detection efficiency is often in the range of $5-20 \%$. The SiPM internal gain is of the order of $10^{5}-10^{6}$, which allows simpler readout electronics compared to conventional gain-less photodiodes.

\subsection{Detector designs}

The first detector design used dual $3 \times 3 \mathrm{~mm}$ SiPMs coupled via light guides to opposite thin sides of a $15 \times 15 \times 2 \mathrm{~mm}$ EJ-204 plastic scintillator tile. The prototype discussed in this paper used SensL $3.16 \times 3.16$ $\mathrm{mm}(2.85 \times 2.85 \mathrm{~mm}$ active area) SPMMicro3035 SiPMs. The light guides, tile edges and top face were painted with Saint-Gobain BC-620 diffusive reflector paint. This design was previously characterised for energy resolution, linearity and uniformity using a proton microbeam from the ANTARES Heavy Ion Microprobe at ANSTO [5, 8]. It was found that detector energy resolution and lowest detectable energy was being limited, in large part, by poor light collection efficiency.
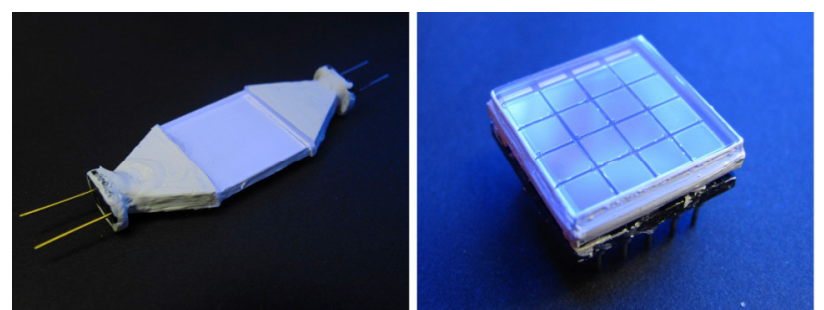

Fig. 1. The dual SiPM (left) and SiPM array (right) prototypes.

The new design aims to improve light collection by increasing the active area presented by the photo detector to the scintillator. The intention is to reduce the proportion of scintillation photons absorbed in the reflective paint or lost through the un-painted surface. A SensL SPMArray4 large area SiPM array was directly coupled to the top, square face of the tile. The SPMArray 4 is a $4 \times 4$ matrix of SPMMicro3035 SiPMs in a $15.81 \times 15.31 \mathrm{~mm}$ ceramic package. The tile edges were coated with BC-620 paint.

As both detectors use the same model of 1 SiPM, when operating at the same bias above breakdown (overbias voltage) the photo-detection efficiency and gain should be approximately equal. Differences in signal between the two prototypes may then be attributed primarily to their different LCE.

\subsection{Electronic readout and processing}

Two FEMTO HVA-500M-20-B preamplifiers with DC $-500 \mathrm{MHz}$ bandwidth and 10x voltage gain were used to pre-amplify the SiPM pulses. The SiPMs were operated at the recommended $2 \mathrm{~V}$ over-bias. The biasing and readout electronics are shown in figure 2. The dual SiPM detector had each SiPM connected to a separate preamplifier. The SPMarray4 detector had 8 SiPMs connected in parallel to each of the preamplifier inputs. The SiPMs were alternately mapped to the two channels to form a checkerboard arrangement. In this setup, the amplifier outputs provide a voltage signal corresponding to the superposition of SiPM current pulses flowing through the $50 \Omega$ input impedance.
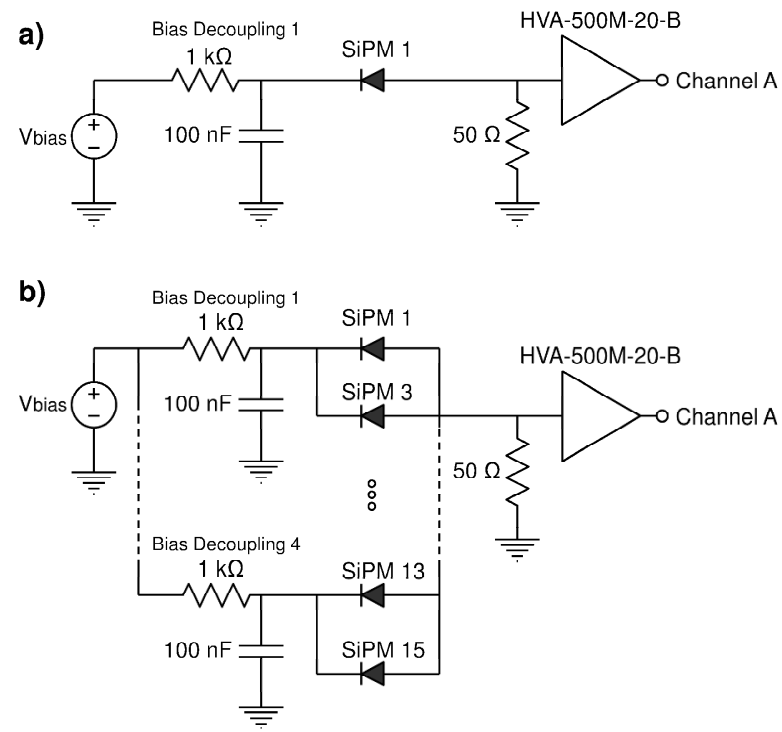

Fig. 2. Readout electronics for a single pre-amplification channel in the (a) dual SiPM and (b) SiPM array prototypes.

The pre-amplified signals were digitized using a PX14400 400 MSps $200 \mathrm{MHz}$ bandwidth PCIe digitizer for the DT fast neutron measurements and a $5 \mathrm{GSps}$ $200 \mathrm{MHz}$ bandwidth DRS-4 based USB digitizer for the uniformity experiment [9].

The amplitudes of pulses were calculated offline, using in-house software to read the binary files and numerically integrate the area above the baseline of each pulse. This area is proportional to the charge in the current pulse.

\section{Results obtained with DT neutrons}

To compare the relative LCE of the two detector designs, the tiles were irradiated with $14 \mathrm{MeV}$ fast neutrons from a Thermo Fisher A-325 deuterium-tritium (DT) neutron generator with an A-3062 tube. The spectrum endpoint corresponds to the full $14 \mathrm{MeV}$ neutron energy being deposited in the scintillator tile, producing a light yield equivalent to a $7 \mathrm{MeV}$ electron. By assessing the endpoint of spectra collected using each detector, the relative light collection may be estimated. 
The preamplifier signals were digitized by a PX14400 digitizer running in segmented mode and internally triggered on one channel. The DT spectrum for each prototype was collected for 16 minutes. Triggers associated with SiPM dark noise were rejected during data post-processing using anti-coincidence rejection [10].

\subsection{Results}

The energy spectra, reconstructed from the summed signals from both channels are displayed in figure 3 . The DT endpoints were arbitrarily fixed at the lower threshold where the integral (cumulative) count rate falls below $0.1 \mathrm{cps}$. The endpoints for the dual SiPM and SiPM array prototypes were 7,800 and 74,000 respectively (arbitrary units). The SiPM array design tile provides a 9.5 -fold improvement in LCE.

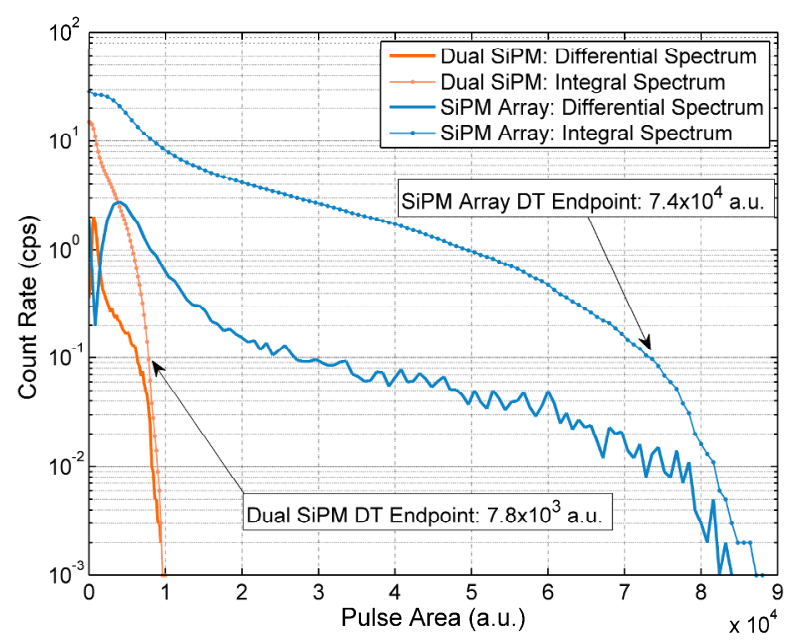

Fig. 3. DT Fast Neutron Spectra measured using the two detector prototypes.

\section{Uniformity test with collimated UV LED}

The uniformity of response with respect to position is an important characteristic of a detector used for spectroscopy. In this particular detector, it is expected that there may be some non-uniformity due to varying light collection efficiency across the face.

Previously, a proton micro-beam was used to measure the response of the dual-SiPM detector in a number of locations across its face [5]. This measurement could be further extended by scanning the micro-beam to build a complete map of detector spatial response. For an initial assessment, a collimated UV light source was used to excite the scintillator at different positions across its face.

\subsection{UV excitation of plastic scintillator}

Scintillators exhibit both an absorption spectrum and a corresponding emission spectrum at longer wavelengths, with some overlap between the two. A $365 \mathrm{~nm}$ UV LED was chosen to excite scintillation from the tile. A $240-400 \mathrm{~nm}$ band-pass filter was placed in front of the
LED to filter out fluorescent light emitted from the epoxy lens.

The tile transmission was measured using the $365 \mathrm{~nm}$ LED to confirm that the UV was being fully absorbed in the $2 \mathrm{~mm}$ thickness. This was to ensure that the UV light was not penetrating through the scintillator and being detected directly by the SiPM array. The LED emission was measured with and without the $2 \mathrm{~mm}$ of EJ-204 in place with an Avantes AvaSpec spectrometer; results are shown in figure 4 . The UV penetration was deemed insignificant in comparison to the intensity of the longer wavelength scintillation light.

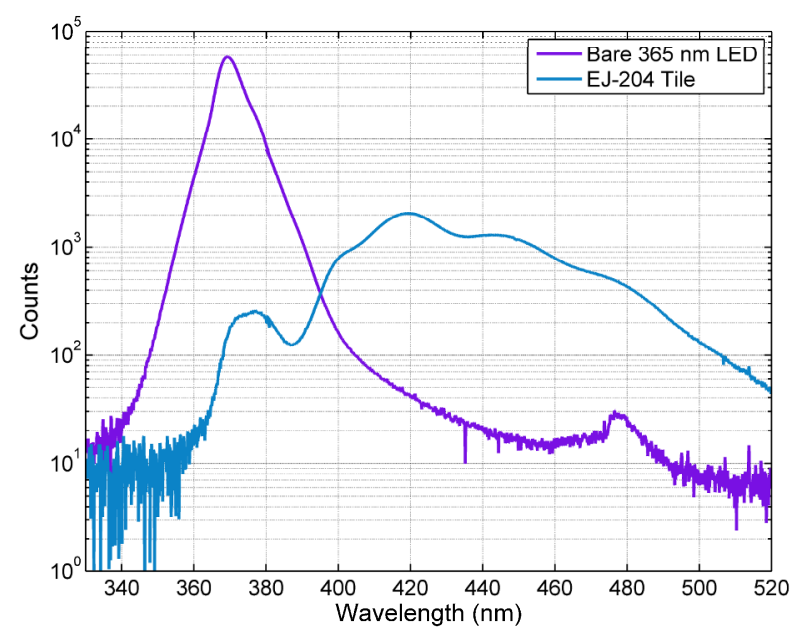

Fig. 4. $365 \mathrm{~nm}$ UV transmission through $2 \mathrm{~mm}$ thick EJ-204.

\subsection{UV scan of tile}

A UV scanning apparatus was constructed using a $365 \mathrm{~nm}$ UV LED mounted within a metal enclosure illuminating a $250 \mu \mathrm{m}$ diameter pinhole at a distance of $8 \mathrm{~cm}$. The UV LED was driven with $15 \mathrm{~ns}$ wide pulses, with 2 ns rise and fall times, from an Agilent 81110A pulse generator. The metal enclosure was attached to an optical table with an $\mathrm{x}-\mathrm{y}$ translation stage. The detector to be tested was mounted on a fixed stage $1 \mathrm{~mm}$ beneath the UV collimator.

The pre-amplified pulses were digitized using the DRS-4 digitizer. The digitizer was triggered externally by the pulse generator synchronisation signal. Software was written to control the digitizer acquisition, process the pulses and record an intensity spectrum for each scan position across the detector face. Once the tile was scanned, a Gaussian fit of each spectrum was performed and the mean intensity determined.

\subsection{Uniformity results}

The spatial response of each prototype is displayed as a contour map in figure 5 and figure 6 . Both detectors exhibit a degree of non-uniformity. The uniformity was quantified as the relative standard deviation of the response measurements across the scintillator face.

The dual SiPM design showed a $7 \%$ non-uniformity. There is an enhanced response when the excitation occurs close to the waveguides. 


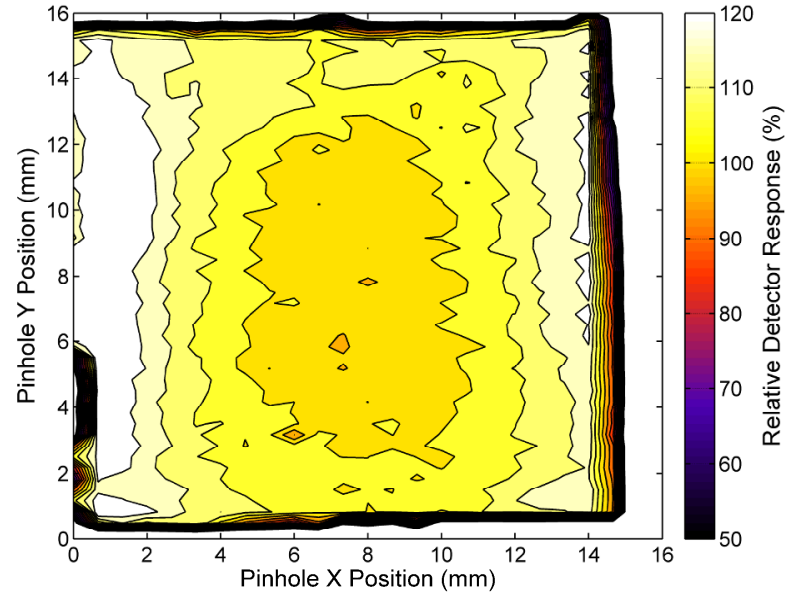

Fig. 5. Spatial response of dual SiPM detector. The light guides are attached on the left and right edges of the plot.

The SiPM array detector had a greater non-uniformity of $13 \%$. The response of the SiPM array detector falls off towards the edges with the minimum response in each corner.

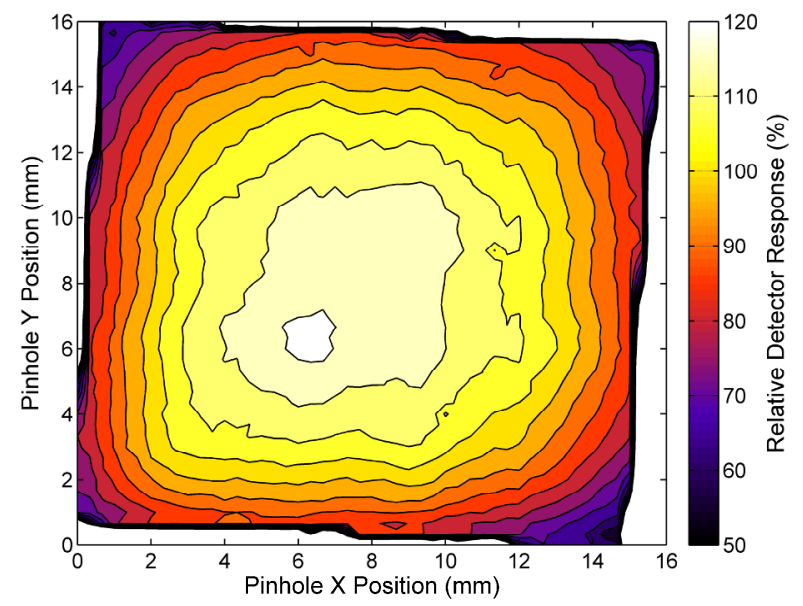

Fig. 6. Spatial response of SiPM array detector.

\subsection{Possible uniformity improvements}

The non-uniformity in both designs is significant enough to degrade the overall energy resolution. As such, methods to improve or correct this are of interest.

With the SiPM array detector, it may be possible to partially improve the uniformity by applying weights to each of the SiPM outputs. To investigate this, the uniformity test will be re-run using 3 preamplifier channels to digitize the edge, corner and centre SiPM groups separately. Various weighting schemes may then be trailed in post processing.

Ultimately, in the multi-layer neutron tracker, the Timepix layer measures the position and direction of the incoming proton. The impact point for a $3 \mathrm{MeV}$ proton can be determined with $1 \mu \mathrm{m}$ precision by fitting the measured cluster with a numeric model that accounts for the proton ionization profile (Bragg peak) and charge sharing between pixels [1]. In such case, the nonuniformity may be corrected event-by-event using the location of the scintillation. This would require a map of response to correct the measured energy. The above UV scanning technique may be used for this; however a proton micro-beam allows a better spatial resolution due to a smaller spot size.

\section{Conclusions and future work}

Two designs of thin scintillator tile detectors with SiPM optical readout were built and characterised for their relative performance. Irradiation with $14 \mathrm{MeV}$ fast neutrons show that the new design, using a large area array of SiPMs, provides a near ten-fold improvement in scintillation light collection efficiency.

A collimated UV LED was used to map the response of the detector across the scintillator face. Both designs exhibited a degree of non-uniformity, with a standard deviation in response of $7 \%$ for the dual SiPM design and $13 \%$ for the SiPM array. Methods to correct for this nonuniformity will be investigated.

\section{Acknowledgments}

This work has been carried out in the framework of the CERN Medipix Collaboration.

\section{References}

1. J. Jakubek, J. Uher, Nuclear Science Symposium Conference Record (IEEE, 2009)

2. J. Jakubek, J. Uher, P. Soukup, Nuclear Science Symposium Conference Record (IEEE, 2010)

3. X. Llopart, R. Ballabriga, M. Campbell, L. Tlustos, W. Wong, Nucl. Instrum. Meth. A 581, 485 (2007)

4. J. Uher, J. Jakubek, Nuclear Science Symposium Conference Record (IEEE, 2010)

5. R. Preston, J. Jakubek, D. Prokopovich and J. Uher, JINST 7, PO2007 (2012)

6. E. Garutti, JINST 6 C10003 (2011)

7. P. Finocchiaro, L. Cosentino, M. Belluso, S. Billotta, G. Bonanno and S. Di Mauro, IEEE Trans. Nucl. Sci. 56, 3 (2009)

8. R. Siegele, D.D. Cohen, N. Dytlewski, Nucl. Intrum. Meth. B 158, 31 (1999)

9. S. Ritt, R. Dinapoli, U. Hartmann, Nucl. Instrum. Meth. A 623, 486 (2010)

10. R. Bencardino, J.E. Eberhardt, R. Preston, Nucl. Instrum. Meth. A 619, 497 (2010) 